\title{
Objeto de Aprendizagem integrado a uma plataforma de educação à distância: a aplicação do COMVIA na UFRGS
}

Profa. Dra. Patricia Alejandra Behar, Universidade Federal do Rio Grande do Sul UFRGS, pbehar@terra.com.br

Msc. Maira Bernardi, UFRGS, mairaber@terra.com.br

Ana Paula Frozi de Castro e Souza, UFRGS, nanafrozi@ yahoo.com.br

Resumo. O COMVIA, é um objeto de aprendizagem sobre COmunidades VIrtuais Aprendizagem, baseado em pressupostos interacionistas. O principal objetivo do COMVIA é uma participação ativa do estudante sobre a construção de seu conhecimento. Este objeto propõe atividades que exploram diferentes recursos tecnológicos que podem ser utilizadas em diferentes áreas. Este estudo também descreve as suas etapas de desenvolvimento, considerando os aspectos técnicos, educacionais e estéticos. O COMVIA foi utilizado na Universidade Federal do Rio Grande do Sul, especificamente em uma disciplina de pós - graduação. Para tanto, este foi integrado a uma plataforma de Educação a Distância, utilizada pela instituição. A aplicação do COMVIA aconteceu no primeiro semestre de 2007. Os dados foram capturados utilizando a plataforma, através dos suas funcionalidades. Neste artigo são descritos os resultados deste estudo, o qual trata sobre a maneira que os alunos interagem com o COMVIA em situações de aprendizagem à distância. Também as principais características dos objetos de aprendizagem e como podem ser integrados a plataforma virtuais.

Palavras-chave: Objetos de aprendizagem, Comunidades Virtuais de Aprendizagem, Educação a Distância.

\section{Integrated Learning Object and Virtual Environment of learning: the application of COMVIA in UFRGS}

\begin{abstract}
COMVIA is a learning object that cares about virtual learning communities based on standard interaction. The main goal of this object is an active participation of the student on his knowledge building. This learning object proposes activities that explore different technological resources that can be employed in different areas. This study also describes the steps to development a learning object, considering educational, aesthetic and technological characteristics. The COMVIA has been used in Universidade Federal do Rio Grande do Sul, specially in Post Graduation Course. It is integrated in a Virtual Learning Environment used by that university institution. The large usage of COMVIA has been on first semester of 2007. The data was captured using the platform, through its tools. In this paper we described the finally results of this study that treats about the way that the students interact through the COMVIA object in e-learning situations. Also its present the main characteristics of a learning objects and how can be integrated into a virtual platform.
\end{abstract}

Key words: Learning Objects, Virtual Learning Communities, e-learning 


\section{Introdução}

O presente artigo trata de uma reflexão acerca dos referenciais teóricos do objeto de aprendizagem (OA) COMVIA $^{1}$, suas etapas de construção e sua aplicação para testes e validação em uma disciplina de pós-graduação ${ }^{2}$ que resultou na formação de comunidades virtuais de aprendizagem. Este é um objeto sobre COMunidades VIrtuais de Aprendizagem (CVA's), projetado por uma equipe interdisciplinar. Na construção do COMVIA, procurou-se atender aos requisitos tecnológicos (suporte computacional), epistemológicos (processo de construção de conhecimentos), metodológicos (práticas pedagógicas) e visuais (design pedagógico e de interação). O objetivo do uso do COMVIA na referenciada disciplina foi justamente verificar o nível dos recursos do objeto em relação a estes requisitos.

O COMVIA foi usado na Universidade Federal do Rio Grande do Sul, Brasil, nos cursos de graduação e pós-graduação da Faculdade de Educação. Este foi integrado ao ambiente de aprendizagem virtual usado nesta instituição, chamado ROODA ${ }^{3}$. Os dados foram capturados usando a plataforma, através do registro nas suas ferramentas em 2007/1.

Este objeto de aprendizagem faz parte do projeto Instrumentalização em Educação a Distância (EAD) ${ }^{4}$, o qual tem como meta a criação de objetos de aprendizagem que integrem pesquisa, desenvolvimento tecnológico e formação de educadores. Seu objetivo é possibilitar aos seus usuários um aprofundamento de conhecimentos teórico-práticos acerca de temática recorrentes na educação a distância, como Comunidades Virtuais de Aprendizagem, Escrita Coletiva e Projetos. Este projeto compreende que os objetos de aprendizagem (OA's) favorecem uma nova concepção de aprendizagem, apoiada por computador, a qual se caracteriza por promover a construção de conhecimento através da interação. Portanto, acredita-se que o sujeito deva exercer um papel ativo frente a sua aprendizagem, apoiado por objetos que permitem a sua atuação e reflexão sobre os conteúdos trabalhados. Logo, procura-se conceituar o termo objeto de aprendizagem e CVA's, abordando aspectos referentes à construção do COMVIA, bem como, o seu uso integrado a uma plataforma de educação a distância e os resultados desta integração.

\section{Conceituando Objeto de Aprendizagem}

O OA tem como principal característica a possibilidade de reutilização de seus recursos em diferentes contextos. Neste estudo, entende-se por Objeto de Aprendizagem qualquer recurso digital como, por exemplo: textos, animação, vídeos, imagens, aplicações, páginas Web em combinação. Os OA's são recursos autônomos, que podem ser utilizados como módulos de um determinado conteúdo ou como um conteúdo completo. Sua utilização é destinada a situações de aprendizagem tanto na modalidade à distância quanto presencial. Segundo o LTSC ${ }^{5}$, estes podem ser definidos por qualquer entidade, digital ou não digital, que possa ser utilizada, reutilizada ou referenciada durante o aprendizado mediado por tecnologias.

\footnotetext{
${ }^{1}$ Disponível em: http://www.nuted.edu.ufrgs.br/instrumentalizacao_em_ead/comvia

${ }^{2}$ Disciplina SA: Oficinas Virtuais de Aprendizagem dos cursos de pós-graduação em Educação e pós-graduação em Informática na Educação, período de 2007/1.

${ }^{3}$ Rede cOOperativa de Aprendizagem - plataforma de educação a distância utilizada na UFRGS. Disponível em: www.ead.ufrgs.br/rooda

${ }^{4}$ Fomentado pela Secretaria de Educação a Distância da UFRGS, através do edital 05/2005.

${ }^{5}$ Learning Technology Standards Committee - http://ltsc.ieee.org/wg12
} 
O uso de OA's remete a um novo tipo de aprendizagem apoiada pelo computador, no qual o professor passa do seu "tradicional" papel de transmissor de informação para desempenhar um papel de mediador da aprendizagem. O OA, enquanto recurso pedagógico, propicia uma participação ativa do aluno na construção e no seu desenvolvimento cognitivo.

Um objeto de aprendizagem pode auxiliar o professor em sua ação docente, na medida em que oferece diferentes ferramentas que servem de apoio ao processo de aprendizagem. A utilização de elementos multimídia, tais como simulações, imagens, textos, som, animações e vídeos, desempenham um papel importante na aquisição de conhecimento quando bem utilizadas. Estes podem ser considerados como recursos pedagógicos que permitem ao aluno acompanhar o conteúdo de acordo com o seu próprio ritmo, acessando facilmente a informação e se engajando de forma independente e autônoma num aprendizado por descoberta. As ferramentas comunicacionais, como por exemplo: chats, fóruns de discussões, e-mails e blogs potencializam o processo de interação entre sujeitos, aumentando o grau de envolvimento das pessoas e, como conseqüência, elevam o grau de conscientização sobre o próprio processo de desenvolvimento cognitivo e a relação deste com o contexto social.

Para garantir sua reusabilidade e acesso de forma independente do espaço e tempo, destaca-se a importância da utilização do padrão de metadados para seu posterior armazenamento em repositórios de objetos. Este padrão é utilizado para recuperar, reutilizar e combinar diferentes objetos, promovendo também a interoperabilidade. Para isso, o conteúdo é estruturado de forma que cada módulo/conteúdo possa configurar-se como uma unidade independente. Esta possibilidade permite misturar diferentes unidades de aprendizagem e colocá-las juntas para novas finalidades e em novas propostas de aprendizagem. Os metadados descrevem e estruturam a informação registrada sob diferentes suportes documentais, facilitando a localização e descrição deste objeto. Portanto, o COMVIA foi submetido ao padrão $\operatorname{LTSC}^{6}$, a fim de permitir a sua reutilização em outros cursos e por outras instituições.

\section{O Objeto de Aprendizagem COMVIA}

O COMVIA é um OA sobre comunidades virtuais de aprendizagem baseado em pressupostos interacionistas. Esta ferramenta propicia uma participação ativa do aluno na construção e no seu desenvolvimento cognitivo. Sua utilização é destinada em situações de aprendizagem tanto na modalidade à distância como na presencial.

Este objeto tem como principal objetivo orientar o usuário na compreensão e utilização das CVA's em sua prática pedagógica. O COMVIA propõe atividades que exploram diferentes recursos tecnológicos e que podem ser empregados em diferentes campos de atuação, visando a instrumentalização teórica e prática sobre a temática abordada.

Para desenvolvimento deste objeto, consideraram-se tanto os aspectos educacionais quanto estéticos e tecnológicos. Assim, pretende-se oferecer ao usuário uma ferramenta de fácil navegação e agradável experiência estética, de forma que os objetivos educacionais possam ser atendidos.

\footnotetext{
${ }^{6}$ Learning Technology Standards Committee - http://ltsc.ieee.org/wg12
} 


\subsection{Desenvolvimento do COMVIA}

O COMVIA foi desenvolvido em quatro etapas principais: concepção do projeto, planificação, implementação e avaliação, seguindo os critérios necessários para o desenvolvimento de objetos de aprendizagem definidos por Amante \& Morgado (2001). A seguir, descrevem-se os caminhos percorridos para o desenvolvimento do COMVIA, dentro de cada etapa.

\subsubsection{Concepção do projeto}

$\mathrm{Na}$ etapa de concepção do projeto, foram definidas as suas linhas mestras e a aplicação que se pretendia desenvolver a partir da idéia inicial do grupo. Nesta primeira etapa, também foram estabelecidos os pressupostos teóricos deste objeto, bem como seus principais objetivos. A equipe foi definida de acordo com as necessidades expostas pelos objetivos. O grupo interdisciplinar foi composto de três educadoras, que realizaram o planejamento e o projeto do COMVIA e uma designer responsável pela implementação da interface do objeto.

Desta forma, foi possível desenvolver um OA com uma boa aparência estética e de fácil navegação, atendendo ainda às necessidades pedagógicas. Neste primeiro momento foi, também, realizada a caracterização do público-alvo do objeto: alunos de licenciatura e professores em educação continuada. Assim, delimitou-se os conteúdos e os principais objetivos que se pretendia desenvolver no COMVIA, além do tipo de aplicação que seria feita.

\subsubsection{Planificação}

No período de Planificação foi realizada a pesquisa para o seu desenvolvimento, o estudo do storyboard e da navegação, devido à sua estrutura não-linear. Foram pesquisados os recursos deste objeto, bem como os tipos de recursos que disponibilizaria (textos, vídeos, imagens, etc.), a metáfora visual e o desenho da interface.

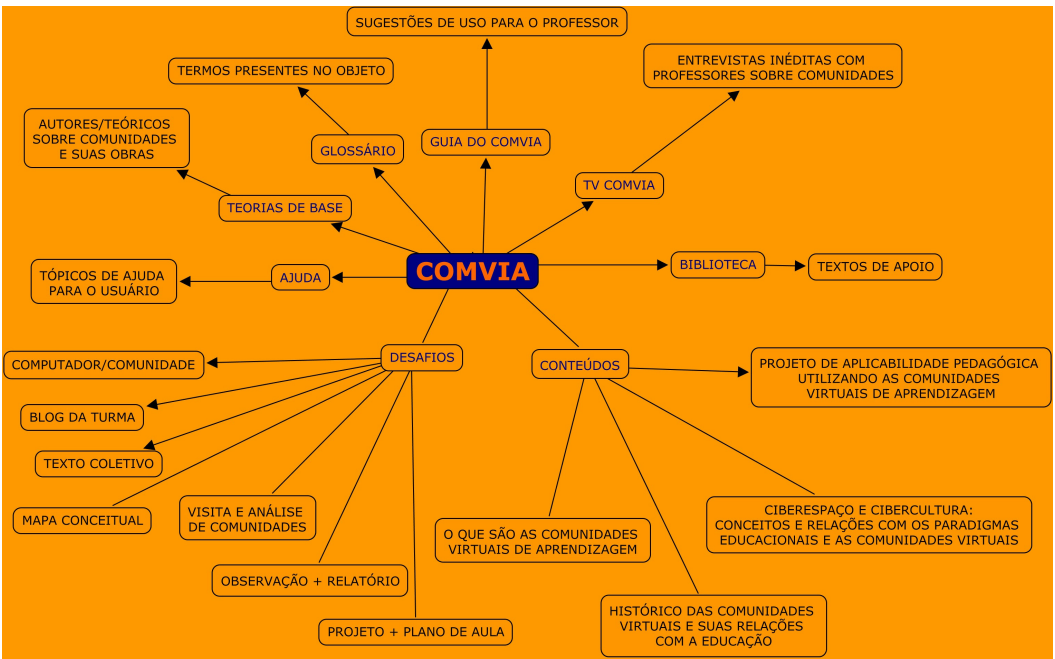

Figura 2. Storyboard com os caminhos da navegação não-linear do objeto COMVIA. 
Para o desenho da interface, foi feito um estudo a partir das definições do papel do design na elaboração de objetos de aprendizagem e como implementar um novo ambiente de ensino no qual o aluno seja o responsável pelo andamento da sua aprendizagem. Para que isso se confirme, é importante tornar o conteúdo dinâmico e destinar-lhe posição de destaque. Quando um sistema interativo é bem desenhado, a interface praticamente desaparece, deixando o aluno concentrado apenas no trabalho, na exploração e no entretenimento. Portanto, o design didático do objeto foi definido em função da intencionalidade e da funcionalidade que se pretendia, identificando possíveis estratégias de aprendizagem e implementando-as de forma dinâmica.

Ainda nesta fase, foi realizada a delimitação dos conteúdos, com um levantamento teórico de material impresso e de textos disponibilizados na web sobre a temática. Também foi necessário constituir uma especificação dos objetivos pedagógicos que o objeto se propunha em atingir a partir do levantamento do públicoalvo, ou seja, educadores e educandos de cursos na área de educação e comunicação. A equipe interdisciplinar reuniu-se para delinear os principais aspectos teóricos que seriam explanados e iniciou a escrita do referencial teórico. Nesta perspectiva, o COMVIA foi pensado de modo que o próprio usuário fizesse o seu caminho de navegação no objeto, tornando-se um agente de sua aprendizagem. Sua metáfora visual foi baseada em jogos de quebra-cabeça, por trazer implícita a idéia do trabalho conjunto e cooperativo de uma comunidade.

\subsubsection{Implementação}

A fase de Implementação refere-se ao desenvolvimento propriamente dito, na qual é definida a ferramenta de programação que será utilizada e são elaborados os primeiros protótipos do objeto até alcançar a sua versão final. O COMVIA foi desenvolvido em Flash7, com animações e recursos interativos.

Foram elaborados três protótipos, com diferentes tipos de animação até a definição da versão final. O primeiro protótipo caracterizou-se dentro de uma estrutura pedagógica linear e fragmentada que não atendia a proposta inicial por estar refletindo uma prática instrucionista de educação. Isto comprometia seu uso dentro de uma proposta mais dinâmica e significativa. Logo, faltava atender os critérios de usabilidade e de uma interface agradável e convidativa ao trabalho.

Já no segundo protótipo, foram realizadas algumas melhorias. A estrutura utilizada em forma de animações possibilitou um avanço em termos de design de interação. Acredita-se que esta proposta estava mais próxima da concepção pedagógica de cunho interacionista. No entanto, outras alterações ainda precisavam ser implementadas e novos recursos oferecidos.

Na terceira versão foi definida a interface e o COMVIA foi finalizado, contando, assim, com oito recursos Dentre os recursos do COMVIA estão: Teorias de Base (referenciais teóricos e biografia de autores como Lévy, André Lemos, Rena Paloff \& Keith Pratt, entre outros), Conteúdos (textos elaborados pela equipe, com base nos referenciais teóricos, sobre temas, sendo intitulados: o que são CVA's, o histórico das CVA's, suas relações com o ciberespaço e a cibercultura e uma reflexão sobre sua aplicação pedagógica), Banco de Desafios (propõe sete desafios que podem ser usados de acordo com a prática pedagógica do professor), TV COMVIA (entrevistas com professores e pesquisadores de educação e comunicação), Biblioteca (outros textos e links interessantes acerca do tema do objeto), Guia do COMVIA (sugestões para o

7 Flash é um programa gráfico vetorial utilizado para criar animações interativas que utiliza a linguagem ActionScript. Esta é a linguagem de programação utilizada em aplicações SWF. 
professor sobre os recursos do COMVIA e suas possibilidades de uso), Glossário (termos específicos do tema CVA's), Ajuda (esclarecimentos sobre os recursos do objeto) e também um agente animado, Comvi, para interagir com o usuário.
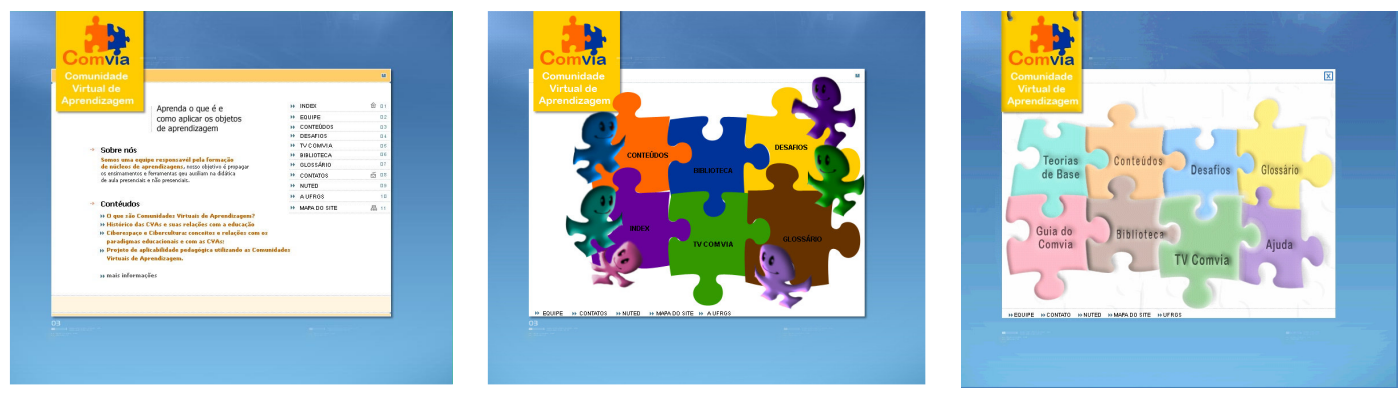

Figura 2. Interface dos protótipos 1, 2 e 3: importantes melhorias realizadas para atender os critérios de usabilidade.
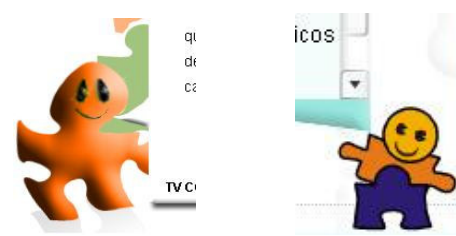

Figura 5. Protótipo 1 e 2 do agente animado Comvi respectivamente.

É importante destacar que no terceiro protótipo houve importantes melhorias nos critérios de usabilidade e design pedagógico do COMVIA, como por exemplo, o menu lateral que permite o acesso aos outros recursos do objeto, sem precisar voltar ao menu inicial, mantendo todos os botões no mesmo lugar a fim de facilitar a navegação. $\mathrm{O}$ mesmo ocorre com o menu superior, que apresenta o conteúdo de cada recurso.

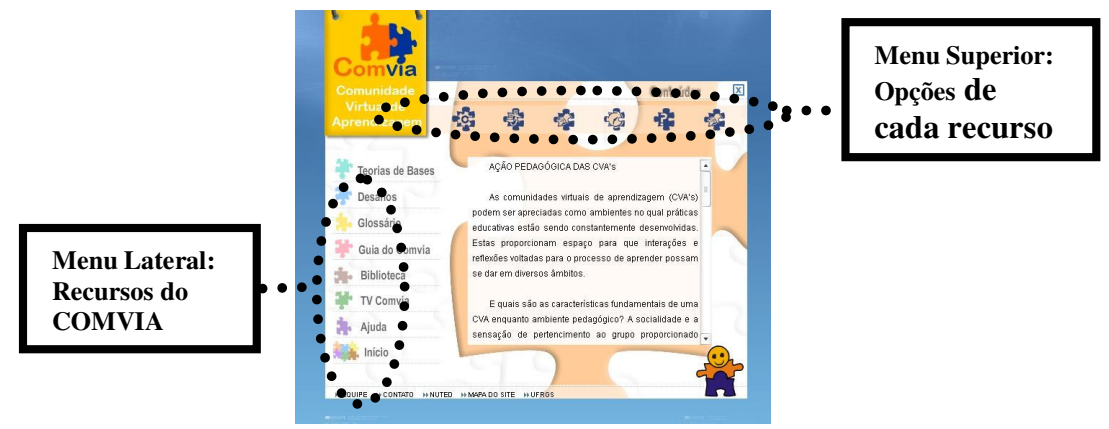

Figura 6. Interface do recurso "Conteúdos" do objeto COMVIA, respeitando os critérios de usabilidade e de design pedagógico.

\subsubsection{Avaliação}

Esta fase consiste em testar o funcionamento do objeto, seu grau de adequação ao público-alvo e o nível de cumprimento dos objetivos. Foram realizados testes para verificar o funcionamento do COMVIA, em uma disciplina de graduação da Faculdade de Educação Também foi analisado se o objeto estava de acordo com características técnicas (tamanho do arquivo e programação), funcionais (animações e layout das telas) e didáticas (referenciais teóricos e desafios) que constavam no projeto. 
As etapas foram realizadas de forma cíclica, ou seja, passaram por uma análise da programação, design, teste e validação, para que posteriormente fosse realizada a reestruturação dos recursos do COMVIA, até chegarem em um nível de funcionamento estável e ser o mais intuitiva possível para os usuários do objeto. Os testes e a validação do objeto ocorreram em $2007 / 1^{8}$, a qual será descrita no presente artigo. Para a validação do objeto estas disciplinas foram ministradas dentro de uma proposta interacionista, na qual os alunos puderam aprender, principalmente através das trocas de experiências.

\section{A validação do COMVIA no pós-graduação através de uma plataforma de educação a distância}

Em 2007/1, o COMVIA foi aplicado em fase de testes na disciplina SA: Oficinas Virtuais de Aprendizagem, dos cursos de pós-graduação em Informática na Educação e Educação/UFRGS. Esta aborda uma área de conhecimento interdisciplinar que integra a Tecnologia Digital com a Educação, como forma inovadora de uso de ambientes de aprendizagem à distância. O objetivo desta é o estudo, em forma de oficinas a serem realizadas através do ambiente virtual de aprendizagem ROODA.

Nesta disciplina de pós graduação, os alunos tiveram contato com o COMVIA através da oficina Comunidades Virtuais de Aprendizagem - COMVIA. Esta oficina tinha como principais objetivos (1) propiciar um espaço para discussão conceitual acerca das CVA's, relacionando-a às vivências dos alunos em comunidades virtuais, bem como, (2) explorar a diversidade de ferramentas que as comunidades de aprendizagem utilizam, (3) orientar o aluno na compreensão e utilização das CVA's em sua prática pedagógica e (4) explorar a diversidade de ferramentas que as comunidades de aprendizagem oferecem, relacionando com sua prática pedagógica. Fez parte da proposta da oficina propor atividades que explorassem diferentes recursos tecnológicos e que pudessem ser empregados em diferentes campos de atuação, visando a instrumentalização teórica e prática do tema e explorando a diversidade de ferramentas que o objeto oferecia. Portanto, os alunos foram convidados num primeiro momento, a explorar os recursos do OA, dentre eles Teorias de Base, Conteúdos, Biblioteca e Glossário.

Todas as atividades foram realizadas com o apoio do ambiente virtual de aprendizagem ROODA, utilizando as ferramentas Fórum, Webfólio, Diário de Bordo, Aulas, Bate-Papo, Grupos e A2. Foram realizados quatro encontros, sendo três presenciais e um virtual, totalizando 20h/a.

\subsection{Integrando o objeto COMVIA e a Plataforma ROODA}

As oficinas foram estruturadas de modo que o objeto de aprendizagem COMVIA apresentasse os conteúdos e desafios aos participantes de forma dinâmica e interativa, através de desafios, vídeos, textos e links. O ambiente virtual ROODA foi usado para que os alunos tivessem um espaço para realizar suas interações. Para isso foram utilizadas ferramentas de interação assíncronas (Fórum, Diário de Bordo e Grupos), síncronas (Bate-Papo e A2) e para postagem de arquivos (Aulas, Webfólio e Webfólio do Grupo).

Em cada aula foram incentivadas as trocas e o estabelecimento das interações entre os alunos durante discussões acerca das temáticas trabalhadas, por meio do

\footnotetext{
${ }^{8}$ SA: Oficinas Virtuais de Aprendizagem do curso de pós-graduação em Educação e Informática na Educação.
} 
ambiente ROODA. Entende-se que, através das interações vivenciadas, seria possível percorrer caminhos diferenciados rumo à construção de novos conhecimentos. Durante as aulas presenciais, foram discutidos aspectos referentes à concepção, criação e a implicação pedagógica das comunidades virtuais de aprendizagem nos diferentes contextos educativos. Os alunos puderam expor suas impressões, reflexões e dúvidas, reconhecendo o potencial das comunidades virtuais de aprendizagem como recurso a serviço da educação, principalmente na Educação à distância. Nos encontros à distância os grupos se encontravam virtualmente, através do ambiente ROODA com o intuito de resolver os desafios propostos.

Os desafios foram realizados em grupos. Logo, os alunos precisaram se encontrar virtualmente ou presencialmente fora do período de aula. Tal necessidade proporcionou que estes, através das ferramentas do ambiente ROODA, constituíssem um espaço virtual com seu grupo, o que resultou na constituição de espaços de coletividade entre os participantes. Assim, foi possível observar a formação de comunidades virtuais de aprendizagem entre os grupos para a realização dos desafios propostos. Para tanto, os alunos utilizaram basicamente as ferramentas Bate-Papo, A2, Grupos e Fórum. Através destas ferramentas os grupos podiam discutir os conteúdos apresentados em aula e definir as linhas mestras do seu trabalho e como este seria apresentado. O Webfólio do Grupo permitiu que cada participante postasse suas pesquisas e modificações no corpo do trabalho, trabalhando de forma cooperativa.

\subsection{Por que integrar plataformas de EAD e objetos de aprendizagem?}

O uso de uma plataforma de educação a distância (EAD) integrada ao objeto de aprendizagem (OA) foi fundamental para que fosse possível a interação de forma síncrona e assíncrona entre os alunos, através das funcionalidades que esta oferece. Assim, durante o período da disciplina foram discutidos os diversos temas/desafios disponibilizados pelo objeto de aprendizagem e, constituídos espaços de coletividade, permitindo o trabalho cooperativo. Tal possibilidade viabilizou a vivência em uma comunidade de aprendizagem por parte dos participantes, como foi possível observar no decorrer das oficinas. A interação é um processo dialético e complexo de trocas e significações, a partir do qual o sujeito e o objeto se modificam (Behar et al., 2007b). Nesta perspectiva, a ação torna-se o instrumento de troca, construindo conhecimento através dos esquemas e da coordenação de ações. Entende-se, assim, o sujeito como uma totalidade, constituído por fatores internos (maturação) e externos (ações do meio). Este processo interativo permitiu que os próprios alunos sejam mediadores de sua aprendizagem, aprendendo através de um processo de cooperação (Duran \& Vidal, 2007).

Logo, como resultado da aplicação deste objeto de aprendizagem através do ambiente ROODA, foi constituído pelos alunos (juntamente com a equipe de apoio) um momento de reflexão e produção de conhecimento sobre o conceito comunidades virtuais de aprendizagem, registrado através de todo o processo de produção elaborado e publicado pelos grupos de trabalho.

O conceito de comunidade foi desenvolvido, através do próprio objeto COMVIA, o qual apresenta as comunidades como um grupo de sujeitos que estabelecem relações interdependentes, dentro de uma formação social complexa, em suas características específicas e individualizantes (Paloff \& Pratt, 2002). As CVA's configuram-se como redes eletrônicas de comunicação interativa, organizadas em torno de um projeto mútuo. Estas se constituem a partir de interesses comuns de conhecimento, metas compartilhadas e valores de troca, estabelecidos em um processo 
cooperativo. A formação de tais redes pode ser observada no curso, bem como sua manutenção para o estudo de novos conteúdos. Segundo Paloff e Pratt (2002), a comunidade é o veículo através do qual ocorre a aprendizagem. Portanto, também foi preciso promover a autonomia, iniciativa e criatividade de cada grupo, de forma que houvesse uma sensação de pertencimento à comunidade pelos participantes da disciplina. Nesta perspectiva os desafios realizados apontaram para a construção de CVA's através da apresentação de diversas ferramentas que suportam este tipo de ação

Por seu caráter não-linear, o objeto contribuiu para que estas ações não percorressem caminhos determinados a priori, respeitando uma linearidade, mas que fossem construídos por conexões, adições, o que Lemos $(2002$, p.75) define como "um passeio pelo espaço cibernético". Entende-se que essa adequação abrange a formação de um conceito acerca das ações realizadas. $\mathrm{O}$ pretendido não era que os alunos somente realizassem as atividades por meio das ferramentas do ambiente, mas que manifestassem a ciência sobre as suas ações9.

Nesta perspectiva, pôde-se observar que os alunos apropriaram-se das informações disponibilizadas pelo objeto, através da troca vivenciada no decorrer da disciplina. Isto foi possível pois eles mesmos formaram comunidades virtuais de aprendizagem com o suporte das funcionalidades do ambiente ROODA.

Através dos dados registrados na plataforma, também foram levantadas sugestões de aperfeiçoamento para o objeto de aprendizagem em questão, tais como: melhorias no agente animado, para uma interação mais significativa com o usuário, a produção de outros vídeos para a TV COMVIA e correção de erros em links da biblioteca.

\section{Considerações Finais}

Concluindo, pode-se dizer então, que a integração entre o objeto de aprendizagem COMVIA e a plataforma ROODA, permitiu a interação entre os alunos da oficina, constituindo uma coletividade entre eles. Tal coletividade culminou na formação de comunidades virtuais que puderam ser observadas no decorrer dos encontros presenciais e à distância, resultando em produções finais de boa qualidade por parte dos grupos. Verificou-se que a comunicação síncrona e assíncrona acerca do OA não seria possível sem o uso de uma plataforma de educação a distância.

A experiência de validação do objeto também permitiu à equipe de desenvolvimento testar a plasticidade do mesmo no que se refere ao seu conteúdo. Este é um aspecto importante para a possibilidade de reutilização deste, atendendo aos critérios de reusabilidade propostos pelo LTSC. Logo, o presente estudo também permitiu realizar o levantamento de dados sobre as melhorias a serem efetuadas, bem como a validação dos objetivos pedagógicos do objeto. Portanto, pode-se dizer que o COMVIA permite o acesso à informação e à realização das atividades de forma independente e autônoma num aprendizado por descoberta. A utilização de elementos multimídia, tais como: simulações, imagens, textos, som, animações e vídeos desempenharam um papel importante na aquisição de conhecimento por parte dos alunos. Isto fez com que ocorressem interações e reflexões voltadas para o processo de aprender, constituídas em diversos âmbitos.

Assim, o COMVIA é uma ferramenta ao alcance de professores em formação, alunos e demais usuários da web. Desta forma, acredita-se que este objeto possa auxiliar na instrumentalização teórica e prática sobre a sua temática, através de diferentes

\footnotetext{
${ }^{9}$ Entende-se a apropriação das TIC à luz das reflexões levantadas por Lemos (2002), ao considerar esta como uma forma de utilização, de domínio técnico e também de aprendizagem.
} 
recursos tecnológicos, que podem ser empregados em diferentes campos de atuação. Para tanto, este objeto foi publicado no repositório de objetos de aprendizagem CESTA10. Tal repositório permite a publicação e reutilização de objetos de aprendizagem por parte de qual quer usuário em qualquer curso. Logo, o COMVIA poderá ser usado de forma autônoma ou ainda, para constituir uma unidade maior através da integração de mais módulos.

\section{Referências bibliográficas}

ALAVA, Séraphin e colaboradores. Ciberespaço e formações abertas: rumo a novas práticas educacionais. Porto Alegre: Artes Médicas, 2002.

ALTOÉ, Anair. COSTA, Maria Luisa F. TERUYA, Teresa K. (orgs.) Educação e novas tecnologias. Maringá: Editora EDUEM, 2005.

AMANTE, Lúcia. MORGADO, Lina. Metodologia de concepção e desenvolvimento de aplicações educativas: o caso dos materiais hipermídia. In: Discursos. Lisboa, Portugal. [III Série, número especial]: 27-44, junho, 2001.

BEHAR, P. A. ; SOUZA, Ana Paula Frozi de Castro e ; BERNARDI, Maira (2007a). Comunidades Virtuais de Aprendizagem: a experiência com um objeto de aprendizagem. In: International Conference - Interactive Computer Aided Blended Learning, 2007, Florianópolis. Anais do.. CEFET : Kassel University Press, v. 1.

BEHAR, P. A. ; LEITE, Silvia Meirelles ; BORDINI, Sandra ; SOUZA, Lúcia Barros De ; SIQUEIRA, Luciano Goularte (2007b). Avaliação de Ambientes Virtuais de Aprendizagem: O Caso do ROODA na UFRGS. In: Revista Avances en Sistemas e Informática, v. 4, p. 81-100,

DURAN, David; VIDAL, Vinyet. Tutoria: aprendizagem entre iguais: da teoria à prática. Tradução Ernani Rosa. - Porto Alegre: Artmed, 2007.

LEMOS, André. Cibercultura: tecnologia e vida social na cultura contemporânea. Porto Alegre: Sulina, 2002.

MORÁN, José Manuel (org.). Novas tecnologias e mediação pedagógica. São Paulo: Papirus, 2000.

PALOFF, Rena M. e PRATT, Keith. Construindo comunidades de aprendizagem, no ciberespaço: estratégias eficientes para salas de aula on-line. Porto Alegre: Artmed, 2002.

O Aluno Virtual: um guia para trabalhar com estudantes on-line. Porto Alegre: Artmed, 2004.

POZO, Juan Ignácio. Aprendizes e mestres: a nova cultura da aprendizagem. Porto Alegre: Artmed, 2002.

RAMAL, Andréa C. Educação na cibercultura: hipertextualidade, leitura, escrita e aprendizagem. Porto Alegre: Artmed, 2002.

\footnotetext{
${ }^{10}$ Projeto CESTA - Coletânea de Entidades de Suporte ao uso de Tecnologia na Aprendizagem. Disponível em: http://www.cinted.ufrgs.br/CESTA/
} 